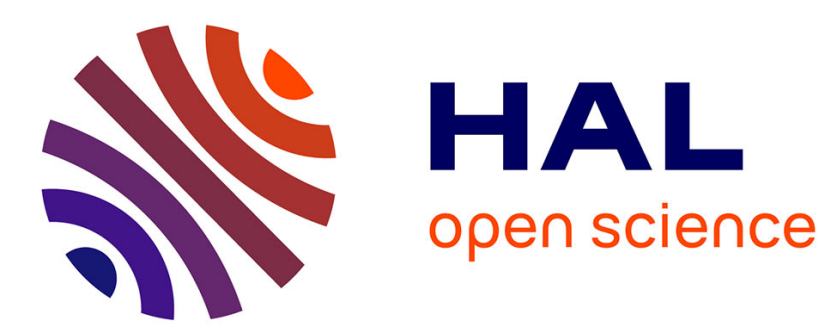

\title{
Stable model order reduction method using Kautz functions: application to VLSI circuits
}

\author{
Mihai Telescu, Pascale Bréhonnet, Noël Tanguy, Pierre Vilbé
}

\section{To cite this version:}

Mihai Telescu, Pascale Bréhonnet, Noël Tanguy, Pierre Vilbé. Stable model order reduction method using Kautz functions: application to VLSI circuits. 11th IEEE Workshop on Signal Propagation on Interconnects, May 2007, Camogli (Genova), Italy. pp.81-84, 10.1109/SPI.2007.4512216 . hal00438757

\section{HAL Id: hal-00438757 \\ https://hal.univ-brest.fr/hal-00438757}

Submitted on 4 Dec 2009

HAL is a multi-disciplinary open access archive for the deposit and dissemination of scientific research documents, whether they are published or not. The documents may come from teaching and research institutions in France or abroad, or from public or private research centers.
L'archive ouverte pluridisciplinaire HAL, est destinée au dépôt et à la diffusion de documents scientifiques de niveau recherche, publiés ou non, émanant des établissements d'enseignement et de recherche français ou étrangers, des laboratoires publics ou privés. 


\title{
Stable model order reduction method using Kautz functions: application to VLSI circuits
}

\author{
Mihai Telescu, Pascale Bréhonnet, Noël Tanguy, Pierre Vilbé \\ Laboratoire d'Electronique et des Systèmes de Télécommunications (LEST) UMR CNRS n6165 \\ 6 Av Le Gorgeu CS93837-29238 BREST Cedex 3 FRANCE \\ Pascale.Brehonnet@univ-brest.fr.
}

\begin{abstract}
In previous papers we presented MOR (Model Order Reduction) methods based on the construction of a Gram matrix subsequent to a decomposition in Laguerre series. In this paper we propose an alternative solution using Kautz series, more adequate for modeling poorly damped systems.
\end{abstract}

\section{Introduction}

Efficient reduced order modeling techniques have become a valuable tool for system designers in recent years, particularly in the field of VLSI circuit interconnects. In previous papers $[1,5,6]$ we presented a Laguerre-Gram reduction method and its applications to VLSI circuits. The Laguerre-Gram method is basically a two step algorithm. The first step supposes a decomposition of the original transfer function (or transfer functions in the MIMO case) in a Laguerre series. With the Laguerre spectrum (or spectra) made available an approximation basis is constructed by repeated application of an adequate operator and a stable rational model is readily calculated. Some difficulties may however appear if the original system displays a slowly attenuated oscillating behavior. The method described in this paper proves a viable alternative in such cases. It follows the same two-step scenario describe above but it is no longer based on single-pole Laguerre functions but on two-pole Kautz functions more appropriate for modeling resonant systems.

\section{On the computation of Kautz Spectra}

In this section we briefly present an efficient solution for computing Kautz spectra. The results provided by the remarkable theoretical works of Wahlberg and Makila $[7,8]$ have proven vital to our research.

Two parameter Kautz functions subsequently denoted as $\varphi_{m}(t)$, with $m=2 n, 2 n+1$, are defined by their Laplace transforms :

$$
\begin{aligned}
& \hat{\varphi}_{2 n}(s)=\frac{\sqrt{2 b c}}{s^{2}+b s+c}\left(\frac{s^{2}-b s+c}{s^{2}+b s+c}\right)^{n} \\
& \hat{\varphi}_{2 n+1}(s)=\frac{\sqrt{2 b} s}{s^{2}+b s+c}\left(\frac{s^{2}-b s+c}{s^{2}+b s+c}\right)^{n}
\end{aligned}
$$

with $n=0,1,2, \ldots$ and $b, c \in \mathfrak{R}^{+^{*}}$.
Note that the orthonormal set $\left\{\varphi_{m}\right\}_{m \geq 0}$ is complete in $L^{2}[0, \infty[$, thus any finite-energy real-causal signal $g(t)$ can be approximated within any desired accuracy by truncating its infinite expansion

$$
g(t)=\sum_{m=0}^{\infty} c_{m} \varphi_{m}(t)
$$

where $c_{m}$ is the $m+1^{\text {th }}$ Kautz-Fourier coefficient, given by the inner product $c_{m}=\left\langle g, \varphi_{m}\right\rangle=\int_{0}^{\infty} g(t) \varphi_{m}(t) d t$.

As proven in [8] if $\hat{g}(s)$ denotes the Laplace transform of $g(t)$ it can be decomposed in two orthogonal partitions $\frac{\sqrt{c}}{s} \hat{g}^{e}(s)$ and $\hat{g}^{o}(s)$ in the following way:

$$
\hat{g}(s)=\frac{\sqrt{c}}{s} \hat{g}^{e}(s)+\hat{g}^{o}(s)
$$

where $\quad \hat{g}^{e}(s)=\sum_{n=0}^{\infty} c_{n}^{e} \hat{\varphi}_{2 n+1}(s) \quad$ and $\quad \hat{g}^{o}(s)=\sum_{n=0}^{\infty} c_{n}^{o} \hat{\varphi}_{2 n+1}(s)$ with $c_{n}^{e}=c_{2 n}$ and $c_{n}^{o}=c_{2 n+1}$.

Let us now consider the z-transforms $C^{e}(z)=\sum_{n=0}^{\infty} c_{n}^{e} z^{-n}$ and $C^{o}(z)=\sum_{n=0}^{\infty} c_{n}^{o} z^{-n}$

It can be shown that the second order all-pass transformation $z=\frac{s^{2}+b s+c}{s^{2}-b s+c}$ yields the following relations binding the Laplace transform and the z-transform of the Kautz spectrum.

$$
\begin{aligned}
& C^{e}(z)=\sqrt{2 b c} \frac{z}{z-1}\left[\frac{\hat{g}\left(a_{-}(z)\right)-\hat{g}\left(a_{+}(z)\right)}{a_{+}(z)-a_{-}(z)}\right], \\
& C^{o}(z)=\sqrt{2 b} \frac{z}{z-1}\left[\frac{a_{+}(z) \hat{g}\left(a_{+}(z)\right)-a_{-}(z) \hat{g}\left(a_{-}(z)\right)}{a_{+}(z)-a_{-}(z)}\right]
\end{aligned}
$$

where $s=a_{ \pm}(z)$ with

$$
a_{ \pm}(z)=\frac{1}{2}\left(b \frac{z+1}{z-1} \pm \sqrt{\left(b \frac{z+1}{z-1}\right)^{2}-4 c}\right)
$$

are the two solutions of the transformation $z=\frac{s^{2}+b s+c}{s^{2}-b s+c}$.

Relations (4) can easily be used to numerically evaluate the Kautz spectrum of a given function from its Laplace transform by setting $z=e^{-j \theta}$ in (4) with $\theta \in[0,2 \pi[$, keeping in mind 
the definition of the z-transform, the Kautz coefficients $c_{m}$ can then be computed using a FFT or DCT algorithm.

\section{Model order reduction}

Let $\hat{g}(s)$ denote the irrational or high order transfer function of the original system we seek to model. As seen in the previous section it can be represented as a Kautz series $\hat{g}(s)=\sum_{n=0}^{\infty} c_{2 n} \hat{\varphi}_{2 n}(s)+\sum_{n=0}^{\infty} c_{2 n+1} \hat{\varphi}_{2 n+1}(s)$.

We build an array of transfer functions $\Omega=\left\{g_{0}, g_{1}, \ldots g_{r}\right\}$ obtained by successive applications of the operator denoted as $\Lambda$ and defined by:

$$
\begin{aligned}
\hat{g}_{0}(s) & \hat{=} \hat{g}(s) \\
\hat{g}_{i+1}(s) & \hat{=} \Lambda \hat{g}_{i}(s) \\
& \hat{=}\left(\hat{g}_{i}(s)-c_{i}^{e} \hat{\varphi}_{0}(s)-c_{i}^{o} \hat{\varphi}_{1}(s)\right) \frac{D(s)}{D(-s)}
\end{aligned}
$$

with $D(s)=s^{2}+b s+c$.

It should be noted that the operator $\Lambda$ has been constructed in such a way that the Kautz spectrum of $\hat{g}_{i}(s)$ can be calculated iteratively, by simply shifting the spectrum of $\hat{g}_{0}(s)$, in other words:

$$
\hat{g}_{i}(s)=\sum_{n=0}^{\infty} c_{2(n+i)} \hat{\varphi}_{2 n}(s)+\sum_{n=0}^{\infty} c_{2(n+i)+1} \hat{\varphi}_{2 n+1}(s)
$$

Let $\Psi$ denote the Gram matrix of inner products $\psi_{i j}=\left\langle g_{i, g_{j}}\right\rangle$ for $i, j=0,1 \ldots, r-1$ and $\vec{b}$ the vector $\left[\psi_{0, r}, \psi_{1, r}, \ldots, \psi_{r-1, r}\right]^{T}$ where $T$ denotes the transpose.

The next step is to write $\hat{g}_{r}(s)$ as a linear combination of $\hat{g}_{i}(s)(i=0,1, . . r-1)$. The procedure yields a rational approximation $\tilde{\hat{g}}(s)$ of $\hat{g}(s)=\hat{g}_{0}(s)$ having the following expression:

$$
\tilde{\hat{g}}(s)=\frac{\sqrt{2 b} \sum_{i=1}^{r} a_{i} \sum_{j=0}^{i-1}\left(\sqrt{c} c_{2 j}+s c_{2 j+1}\right)[D(s)]^{i-j-1}[D(-s)]^{r+j-i}}{\sum_{i=0}^{r} a_{i}[D(s)]^{i}[D(-s)]^{r-i}}
$$

The $\vec{a}=\left[a_{0}, a_{1}, \ldots, a_{r-1}\right]^{T}$ vector represents the solution to the system of equations given by $\Psi \vec{a}=-\vec{b}$.

\section{Remarks}

1. The model is stable by construction. As for LaguerreGram technique [1], this can be proved by a Lyapunov method.

2. The order of the reduced model as given by (7) is $2 r$ since the order of $D(s)$ is 2 . In fact if $p$ is a pole of the reduced model, then $c / p$ is also one. Therefore, in practice we only keep half of the poles (those corresponding to the largest residues) thus reducing the model given by (7) to an order $r$.

3. Note that for a given reduced order denominator one can easily calculate an optimal numerator minimizing the error norm $\|\hat{g}-\tilde{\hat{g}}\|^{2}$.

4. The method can be extended to MIMO systems. For an original matrix of irrational or high order transfer functions a matrix of reduced order rational functions sharing a common denominator is in this case computed.

5. The non-crucial choice of Kautz parameters $b$ and $c$ can be made according to the procedure described in [3].

6. The method can also be adapted to model systems described by discrete data such as frequency measurements of S or Y parameters. In this case the computation of Kautz spectra need to be done differently, the rest of the algorithm remains unchanged.

\section{Example}

The original model chosen to illustrate this method is the admittance matrix $Y$ of a system of two $0.7 \mathrm{~mm}$ long coupled interconnect lines. The lines are supposed identical and symmetrical and therefore the matrix has four distinct terms denoted $y_{i}, i=1,2,3,4$ for simplicity. The aim is to provide rational approximations sharing a common denominator for the 4 distinct terms.

$$
Y=\left[\begin{array}{llll}
y_{11} & y_{12} & y_{13} & y_{14} \\
y_{21} & y_{22} & y_{23} & y_{24} \\
y_{31} & y_{32} & y_{33} & y_{34} \\
y_{41} & y_{42} & y_{43} & y_{44}
\end{array}\right]=\left[\begin{array}{llll}
y_{1} & y_{2} & y_{3} & y_{4} \\
y_{2} & y_{1} & y_{4} & y_{3} \\
y_{3} & y_{4} & y_{1} & y_{2} \\
y_{4} & y_{3} & y_{2} & y_{1}
\end{array}\right]
$$

Figures 1-4 present the original frequency responses and their order 40 rational approximations (with a common denominator). The quality of the reduced model on a wide frequency band is visible and resonance peaks are well reproduced. Passivity could eventually be enforced $[2,4]$ on this model to allow the determination of an equivalent circuit. 

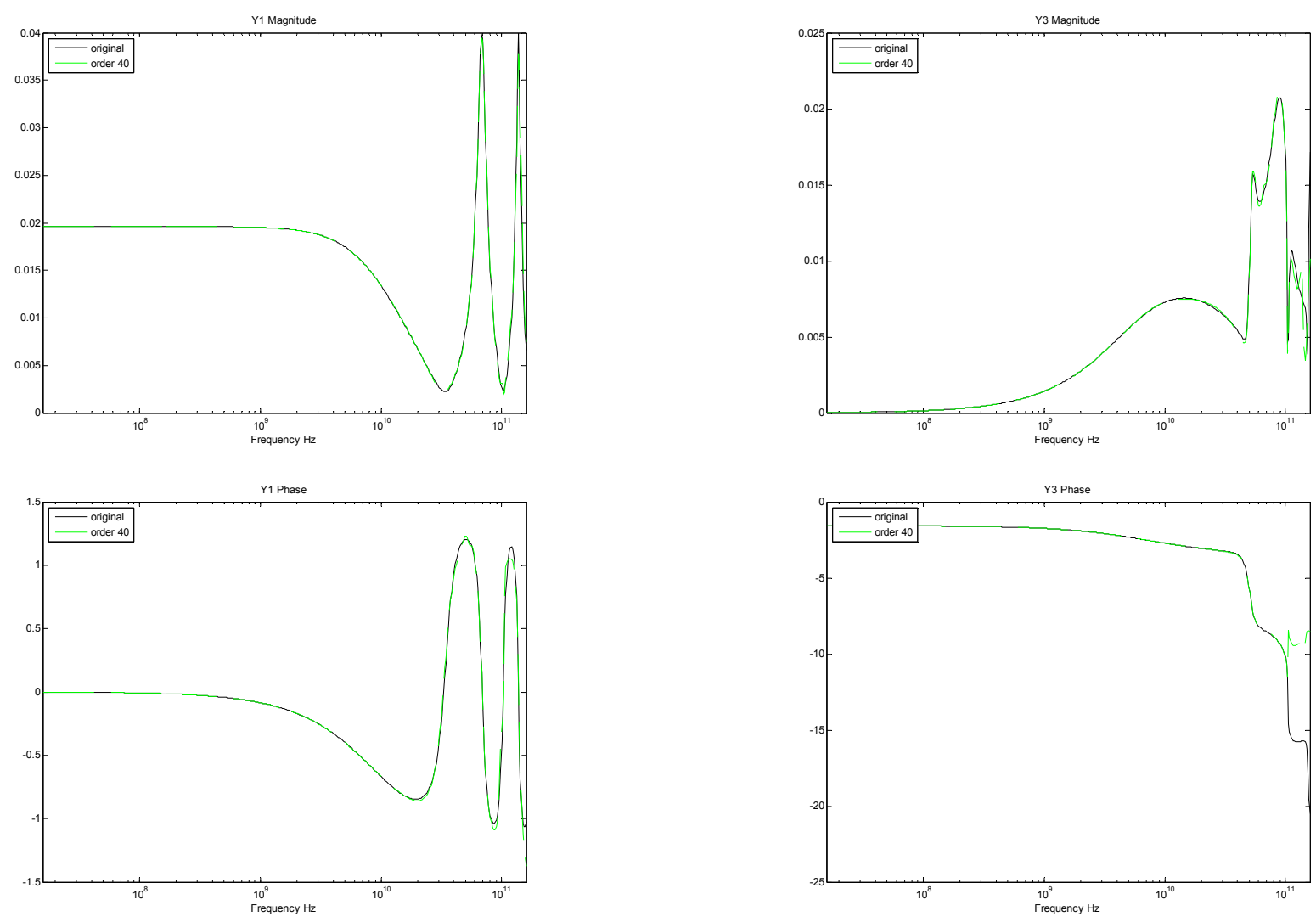

Fig1. $y_{l}$ frequency response

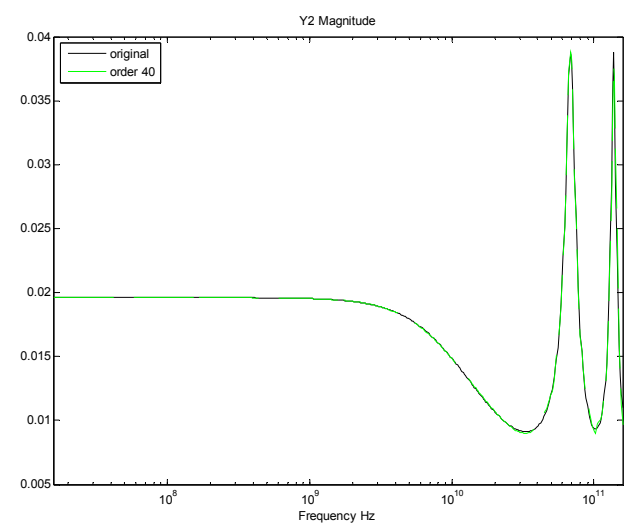

Fig3. $y_{3}$ frequency response
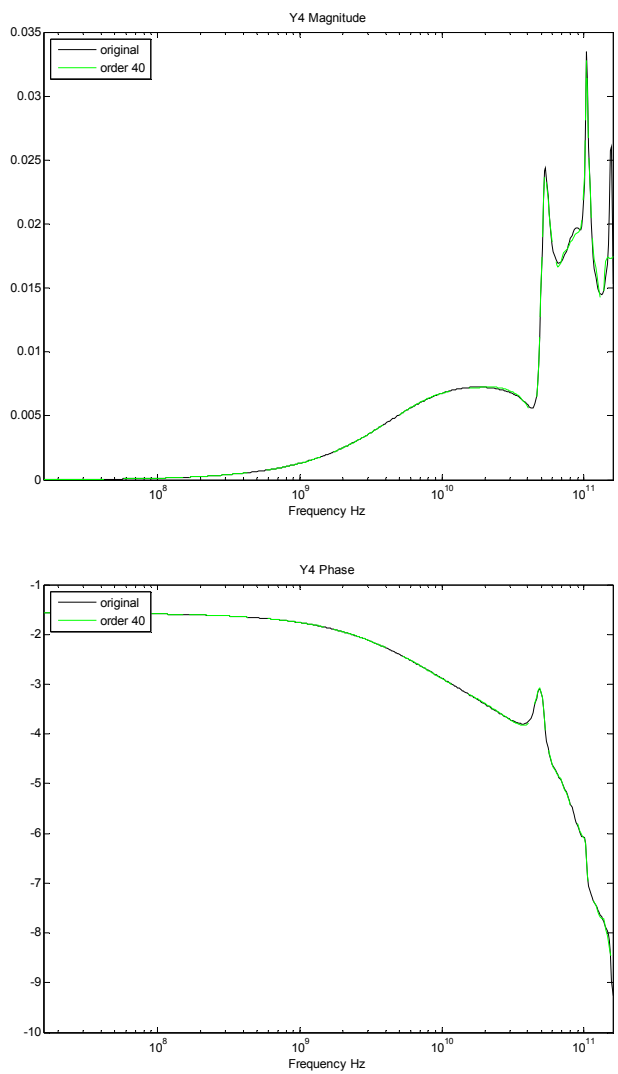

Fig4. $y_{4}$ frequency response

Fig2. $y_{2}$ frequency response 


\section{Conclusions}

A model order reduction method based using Kautz functions was presented. This new technique may present certain advantages when applied to resonant systems. Future research includes testing different operators and refining the algorithm for faster implementation.

\section{Acknowledgements}

The authors thank Brittany region for its financial support.

\section{References}

[1] A. Amghayrir, N. Tanguy, P. Bréhonnet, P. Vilbé, L.C.Calvez, Laguerre-Gram Reduced-Order Modeling, IEEE Transactions on Automatic Control, Vol 50, no 9, pp. 1432-1435, September 2005

[2] B. Gustavsen, A. Semlyen, Enforcing Passivity for Admittance Matrices Approximated by Rational Functions, IEEE Trans. Power Syst., vol. 16, no.1, pp. 97104, February 2001

[3] R. Morvan, N. Tanguy, P. Vilbé, L.C. Calvez, Pertinent parameters for Kautz approximation, Electronics Letters, 36, (8), (2000), 769-771.

[4] S. Grivet-Talocia, Passivity Enforcement via Perturbation of Hamiltonian Matrices , IEEE Trans. Circuits and Systems I: Fundamental Theory and Applications, pp. 1755-1769, vol. 51, n. 9, September, 2004

[5] M. Telescu, P. Brehonnet, N. Tanguy, P. Vilbé, L.C. Calvez, F. Huret, Model-Order Reduction of VLSI Circuit Interconnects via a Laguerre representation, 9th IEEE Workshop on Signal Propagation On Interconnects, Garmisch Partenkirchen, Germany, 10-13 May 2005

[6] M. Telescu, N. Tanguy, P. Brehonnet, P. Vilbé, L.C. Calvez, F. Huret, Low Complexity Equivalent Circuit Models for VLSI Interconnects, 10th IEEE Workshop on Signal Propagation On Interconnects, Berlin, Germany, 912 May 2006.

[7] B. Wahlberg, System identification using Kautz models, IEEE Transactions on Automatic Control, 39, (6), (1994), pp. 1276-1282.

[8] B. Wahlberg, P.M. Mäkilä, On approximation of stable linear dynamical systems using Laguerre and Kautz functions, Automatica, 32, (5), (1996), 693-708. 